\title{
CD147-targeted siRNA in A375 malignant melanoma cells induces the phosphorylation of EGFR and downregulates cdc25C and MEK phosphorylation
}

\author{
MIHO HATANAKA ${ }^{1}$, YUKO HIGASHI ${ }^{1}$, KAZUHIRO KAWAI ${ }^{1}$, JUAN SU $^{2}$, \\ WEIQI ZENG ${ }^{2}$, XIANG $\mathrm{CHEN}^{2}$ and TAKURO KANEKURA ${ }^{1}$

\begin{abstract}
${ }^{1}$ Department of Dermatology, Kagoshima University Graduate School of Medical and Dental Sciences, Kagoshima 890-8520, Japan; ${ }^{2}$ Department of Dermatology, Xiangya Hospital, Central South University, Changsha, Hunan 410008, P.R. China
\end{abstract}

Received November 20, 2014; Accepted June 22, 2015

DOI: $10.3892 / \mathrm{ol} .2016 .4267$

\begin{abstract}
The Raf-MEK-ERK signaling pathway is important during oncogenesis. An activating mutation of BRAF constitutively activates the Raf-MEK-ERK signaling cascade, and has been identified in $\sim 70 \%$ of malignant melanomas (MMs). Cluster of differentiation 147 (CD147)/basigin is an integral plasma membrane protein belonging to the immunoglobulin superfamily. The protein is highly expressed on MM cells, and promotes cellular proliferation and tumor growth. The present study investigated the correlation between CD147 expression and Raf-MEK-ERK signaling in MM using the A375 human MM cell line, which harbors the activating mutation of BRAF. The phosphorylation of epidermal growth factor receptor (EGFR) was upregulated, and mitogen-activated protein kinase kinase (MEK) and cell division cycle 25C phosphorylation was downregulated by CD147 silencing in the A375 cells. Cell growth was inhibited by the EGFR inhibitor erlotinib and by CD147 silencing, and additive growth inhibition was observed when these techniques were combined. The results of the present study indicate that the combination of EGFR and CD147 inhibition may be useful in BRAF-mutated MM.
\end{abstract}

\section{Introduction}

Mitogen-activated protein kinases (MAPKs) mediate intracellular signals transduced by a number of cell surface receptors, including epidermal growth factor receptor (EGFR). Among the MAPK-mediated pathways, the Raf-MEK-ERK signaling pathway has been well characterized and its importance in

Correspondence to: Dr Yuko Higashi, Department of Dermatology, Kagoshima University Graduate School of Medical and Dental Sciences, 8-35-1 Sakuragaoka, Kagoshima 890-8520, Japan

E-mail: higashiy@m.kufm.kagoshima-u.ac.jp

Key words: malignant melanoma, CD147, EGFR, BRAF, erlotinib oncogenesis has been previously demonstrated $(1,2)$. Aberrant activation of the Raf-MEK-ERK pathway is frequently observed in human cancers and contributes to oncogenic properties, such as enhanced cellular proliferation, independent of growth factors and their receptors (3). The activating mutation of BRAF that constitutively activates the Ras-MEK-ERK pathway has been identified in $8 \%$ of all human cancers and in 59\% of malignant melanomas (MMs) (4).

Cluster of differentiation 147 (CD147)/basigin, an integral plasma membrane protein belonging to the immunoglobulin superfamily, is a multipotential molecule expressed widely in various tissues (5-7). The protein is particularly enriched on the surface of malignant tumor cells (8-13), including MM cells (14-17). We previously reported that CD147 expressed on $\mathrm{MM}$ cells played an important role in cellular proliferation and tumor growth in vitro and in vivo $(14,17)$. Our previous in vitro study demonstrated that CD147 promotes the cellular proliferation, invasiveness, metastasis and angiogenesis of MM cells by regulating tumor cell glycolysis (17). CD147 is a multifunctional molecule, and in addition to the regulating of glycolysis, it has a wide range of pathophysiological functions (7). Previous studies have demonstrated that CD147 promotes the growth of human breast carcinoma cells by stimulating hyaluronan production (18) and that it confers resistance to apoptosis (19). In these pathophysiological processes, CD147 directly activates ERK.

The present study investigated the correlation between CD147 and the Raf-MEK-ERK pathway using the A375 MM cell line that harbors the activating mutation of BRAF (20). Considering that CD147 and EGFR promote tumor cell proliferation, it was initially anticipated that EGFR phosphorylation would be reduced by CD147 silencing. However, our preliminary studies identified that CD147 silencing enhances the phosphorylation of EGFR and, therefore, the present study aimed to investigate the molecular mechanism(s) underlying this finding.

\section{Materials and methods}

Cell Culture. The A375 human MM cell line was purchased from the American Type Culture Collection (Manassas, VA, USA). All cells were grown in Dulbecco's modified Eagle's 
medium supplemented with $10 \%$ fetal bovine serum and $1 \%$ penicillin-streptomycin-amphotericin B solution (Invitrogen Life Technologies, Carlsbad, CA, USA). The cells were maintained at $37^{\circ} \mathrm{C}$ in a $5 \% \mathrm{CO}_{2}$ humidified atmosphere.

CD147 silencing. A U6-vshRNA-CMV-PUR lentivirus encoding CD147 short hairpin (sh)RNA (CD147 shRNA lentivirus) was constructed and produced by Shanghai Genechem Co., Ltd. (Shanghai, China) (21). The CD147 shRNA sequences were as follows: Sequence 1, 5'-TGTCGTCAGAACACATCAACT TCAAGAGAGTTGATGTGTTCTGACGACTTTTTTC-3' and sequence 2, 5'-TCGAGAAAAAAGTCGTCAAACACA TCAACTCTCTTGAAGTTGATGTGTTCTGACGACA-3'.

Lentiviral infection. The A375 cells were seeded in 12-well plates in $1 \mathrm{ml}$ of growth medium without antibiotics. Once the cells had reached $70 \%$ confluency (after $\sim 24 \mathrm{~h}$ ), they were infected with $5 \times 10^{8} \mathrm{TU} / \mathrm{ml}$ CD147 shRNA lentivirus or empty lentivirus (control), as described previously (21), for $4 \mathrm{~h}$ at $37^{\circ} \mathrm{C}$. The A375 cells were then grown for 7-10 days in $2 \mathrm{ml}$ of medium containing $400 \mathrm{ng} / \mathrm{ml}$ puromycin (Sigma-Aldrich, St. Louis, MO, USA) and then for $\geq 2$ weeks in medium containing $200 \mathrm{ng} / \mathrm{ml}$ puromycin until stable cell clones could be harvested. Infected cells were selected based on their resistance to puromycin.

Western blot. EGF (100 ng/ml; Wako Chemicals USA, Inc., Richmond, VA, USA) was added to the A375 cell cultures for 0,2,5 and $10 \mathrm{~min}$ to induce EGFR phosphorylation. Following this, total protein was isolated by detaching and suspending the cells in lysis buffer $(150 \mathrm{mmol} / \mathrm{l} \mathrm{NaCl}, 10 \mathrm{mmol} / \mathrm{l}$ Tris, $0.1 \%$ sodium dodecylsulfate, $1.0 \%$ Triton $\mathrm{X}-100,1 \%$ deoxycholates, $5 \mathrm{mmol} / \mathrm{l}$ ethylene diaminetetra acetate) on ice for $30 \mathrm{~min}$ and then centrifuging (4,500 x g for $10 \mathrm{~min})$. Protein concentration was determined using the bicinchoninic acid assay (Pierce Biotechnology, Inc., Rockford, IL, USA) according to the manufacturer's instructions. Equal amounts of proteins were separated by SDS polyacrylamide gel electrophoresis and then electrotransferred to polyvinylidene fluoride membranes. Non-specific antibody binding sites were blocked overnight at $4^{\circ} \mathrm{C}$ in Tris-buffered saline containing $5 \%$ skimmed dry milk and $0.1 \%$ Tween-20. The membranes were subsequently incubated for $1 \mathrm{~h}$ at room temperature (RT) with polyclonal rabbit anti-human CD147 (cat. no. 34-5600; 1:2,000; Invitrogen Life Technologies), polyclonal rabbit anti-human GAPDH (cat. no. GTX100118; 1:500; GeneTex Inc., Irvine, CA, USA), polyclonal rabbit anti-human phosphorylated EGFR (Tyr1068; cat. no. 2234; 1:200; Cell Signaling Technology, Inc., Danvers, MA, USA), monoclonal rabbit anti-human phosphorylated mitogen-activated protein kinase kinase (MEK; cat. no. 9154; 1:1,000; Cell Signaling Technology Inc.), and polyclonal rabbit anti-human phosphorylated cell division cycle 25C (cdc25C; cat. no. 9527S; 1:1,000; Cell Signaling Technology Inc.) primary antibodies. Secondary horseradish peroxidase-conjugated polyclonal goat anti-rabbit IgG antibodies (cat. no. sc-2004; 1:2,000; Santa Cruz Biotechnology Inc., Santa Cruz, CA, USA) were then incubated with the membranes for $30 \mathrm{~min}$ at RT. The membranes were washed three times with Tris-Buffered Saline containing $0.1 \%$ Tween-20 for $5 \mathrm{~min}$. The signal from antibody-conjugated horseradish peroxidase was visualized
A

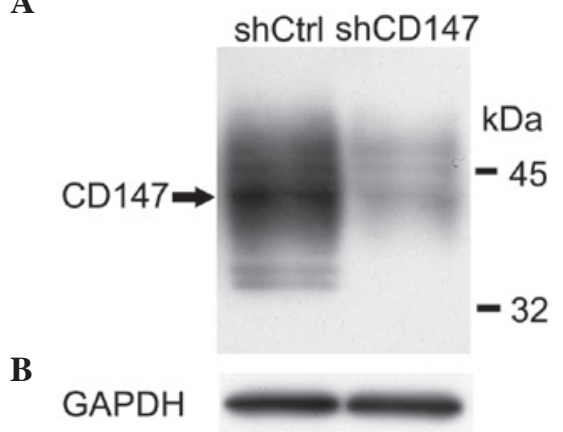

C

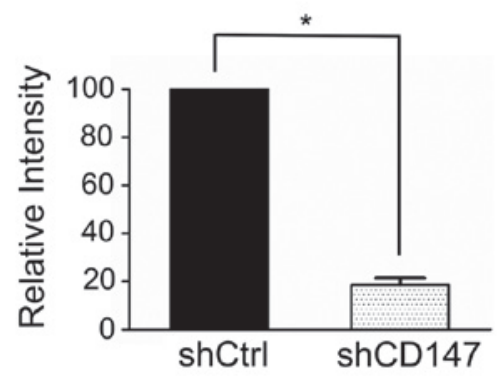

Figure 1. (A) shRNA-mediated silencing of CD147 expression in A375 human malignant melanoma cells. CD147 expression was lower in the A375 cells transfected with CD147 shRNA (shCD147) compared with the control (shCtrl). The CD147 shRNA was delivered using a lentivirus and the control was empty lentiviral vector. (B) GAPDH was used as a loading control. (C) Quantification of CD147 expression levels in shCD147 and shCtrl cells. ${ }^{*} \mathrm{P}<0.05$ vs. control. CD147, cluster of differentiation 147; sh, short hairpin.

by applying SuperSignal ${ }^{\mathrm{TM}}$ West Pico Chemiluminescent Substrate (Thermo Fisher Scientific, Inc., Waltham, MA, USA) and exposing to X-ray film.

Cell growth assay. Cell growth was assayed with the 3-(4,5-dimethylthiazol-2-yl)-2,5-diphenyltetrazolium bromide (MTT) assay. The cells (100 $\mu \mathrm{l}$ at $3 \times 10^{3} / \mathrm{ml}$ per well) were seeded in 96-well flat bottom plates and incubated with $1,000 \mathrm{nM}$ of the EGFR inhibitor, erlotinib (ChemieTek, Indianapolis, IN, USA). At 24, 48, 72 and $96 \mathrm{~h}$, the cells were incubated for $3 \mathrm{~h}$ with $0.5 \mathrm{mg} / \mathrm{ml}$ MTT (10 $\mu \mathrm{l}$ per well). Subsequently, dimethylsulfoxide $(100 \mu \mathrm{l})$ was added to all wells and the plates were kept overnight at RT in the dark. Following this, the absorbance of each well was measured with a microplate reader (iMark ${ }^{\mathrm{TM}}$ microplate absorbance reader; Bio-Rad Laboratories, Hercules, CA, USA). The test and reference wavelengths were 570 and $630 \mathrm{~nm}$, respectively.

Statistical analysis. All data are presented as the mean \pm standard deviation. Differences between two groups were evaluated using Student's t-test. Dunnett's procedure was used for the statistical analysis of differences between multiple groups and the control. All reported $\mathrm{P}$-values are two-tailed, and $\mathrm{P}<0.05$ was considered to indicate a statistically significant difference.

\section{Results}

shRNA-mediated silencing of CD147 expression in A375 cells. The expression of CD147 was knocked down with specific 
A

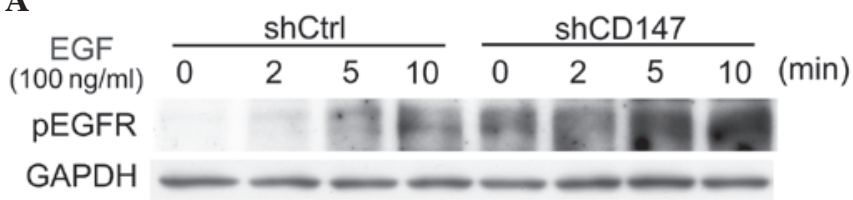

B

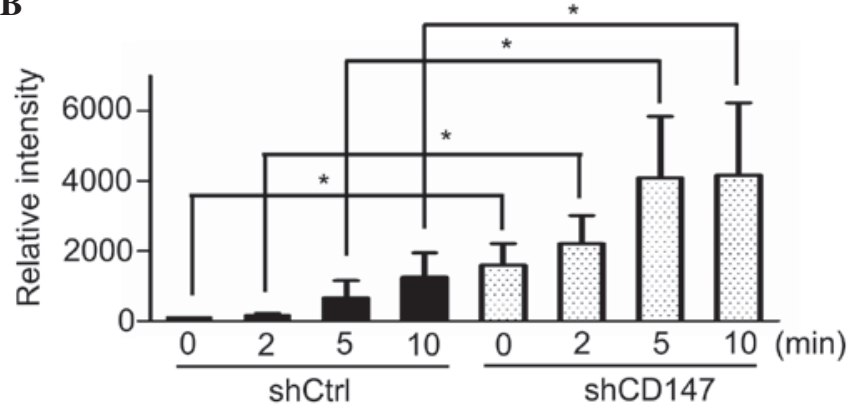

Figure 2. EGFR phosphorylation was induced in A375 human malignant melanoma cells by exogenous EGF $(100 \mathrm{ng} / \mathrm{ml})$ in a time-dependent manner. (A) Western blot analysis revealed that EGFR phosphorylation was upregulated by shRNA knockdown of CD147 (shCD147) compared with the control (shCtrl; empty lentiviral vector). The highest level of EGFR phosphorylation was recorded at $10 \mathrm{~min}$. GAPDH was used as a loading control. (B) Quantification of EGFR phosphorylation levels in shCtrl and shCD147 cells. ${ }^{*} \mathrm{P}<0.05$ vs. control. EGFR, epidermal growth factor; pEGFR, phosphorylated EGF receptor; CD147, cluster of differentiation 147; sh, short hairpin.

shRNA-targeting of CD147 mRNA, and CD147 protein levels were determined using western blotting. CD147 protein levels were decreased in the A375 cells transfected with CD147 shRNA lentivirus (A375 shCD147) compared with the cells transfected with the empty lentivirus control (A375 shCtrl; Fig. 1).

Phosphorylation of EGFR is upregulated by silencing CD147 in A375 cells. The effects of CD147 silencing on EGF $(100 \mathrm{ng} / \mathrm{ml})$-induced EGFR phosphorylation were investigated. The results demonstrated that knockdown of CD147 enhances the phosphorylation of EGFR in a time-dependent manner, when stimulated by its ligand, EGF. The highest level of EGFR phosphorylation was recorded at $10 \mathrm{~min}$ (Fig. 2).

Phosphorylation of MEK and cdc25C is downregulated by silencing CD147 in A375 cells. To investigate the underlying mechanism(s) of CD147-regulated EGFR phosphorylation, the effects of CD147 silencing on the phosphorylation of MEK, a downstream effector of B-Raf was examined. In line with previous studies reporting that CD147 promotes ERK activity $(18,19)$, the results of the present study demonstrated that CD147 was required for high levels of MEK activation, and that MEK phosphorylation was inhibited by CD147 silencing (Fig. 3). As previous studies have demonstrated that ERK phosphorylates cdc25C in Xenopus and that the cde25 homologue, cdc25A, binds to and dephosphorylates EGFR $(24,25)$, the phosphorylation of cdc $25 \mathrm{C}$ was investigated. Fig. 3 shows that the phosphorylation of cdc25C was downregulated by knockdown of CD147 in the present study. confers an additive effect on the reduced growth of A375 cells.
A

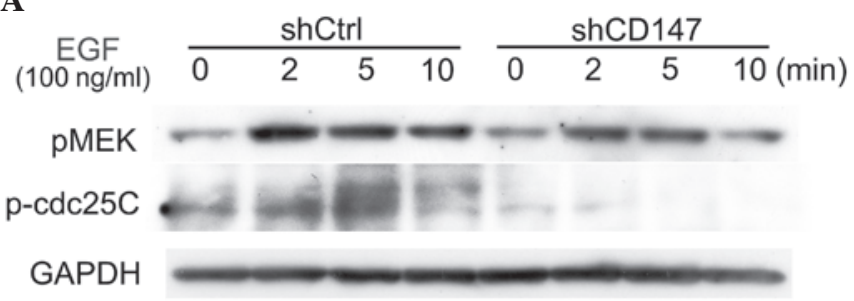

B

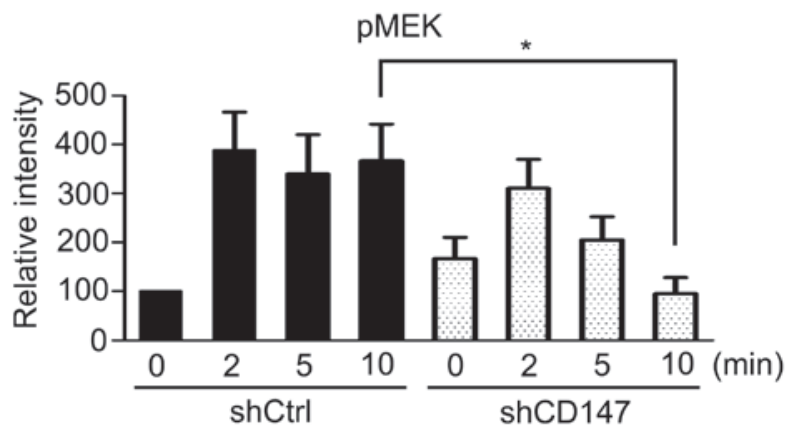

C

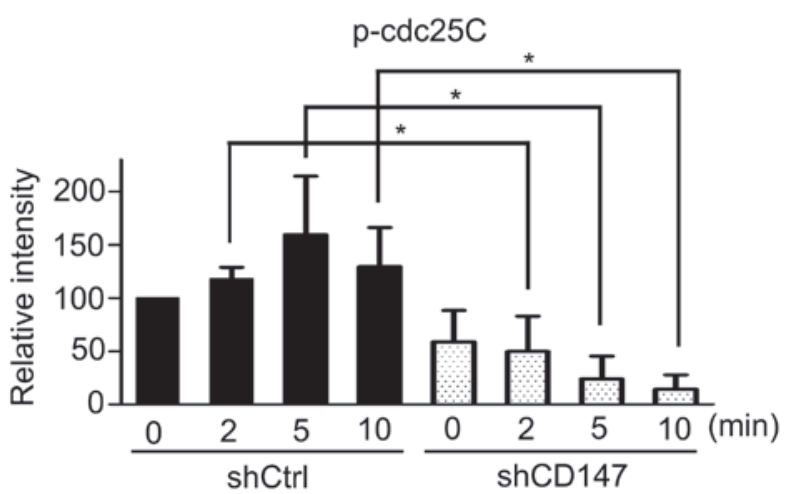

Figure 3. (A) EGFR phosphorylation was induced in A375 human malignant melanoma cells by exogenous EGF $(100 \mathrm{ng} / \mathrm{ml})$ at different time-points. Western blot analysis demonstrates that knockdown of CD147 using lentivirus-delivered shRNA (shCD147) inhibits the phosphorylation levels of (B) MEK and (C) cdc25C, compared with the control (shCtrl; empty lentiviral vector). GAPDH was used as an internal housekeeping control. ${ }^{*} \mathrm{P}<0.05$ vs. control. EGF; epidermal growth factor receptor; pMEK, phosphorylated mitogen-activated protein kinase kinase; CD147, cluster of differentiation 147; sh, short hairpin.

We previously demonstrated that MM cell growth is inhibited by CD147 silencing $(14,17)$, and that EGFR phosphorylation is upregulated by CD147 silencing. To investigate these findings further, the effect of the small-molecule kinase inhibitor of EGFR, erlotinib, on the growth of shCtrl and shCD147 A375 cells was examined. Cell growth inhibition was significantly higher in the shCD147 cells compared with the shCtrl cells $(\mathrm{P}<0.01)$. Growth inhibition by erlotinib was also significantly higher in the shCD147 cells compared with the shCtrl cells $(\mathrm{P}<0.01)$, indicating the additive inhibition of $\mathrm{A} 375$ cell growth by silencing CD147 and treatment with erlotinib (Fig. 4).

\section{Discussion}

MM, a highly aggressive cutaneous tumor, is characterized by rapid growth and a high potential for invasiveness and metastasis. An activating mutation of the BRAF oncogene (V600E) is observed in $\sim 70 \%$ of primary MM (4). The 


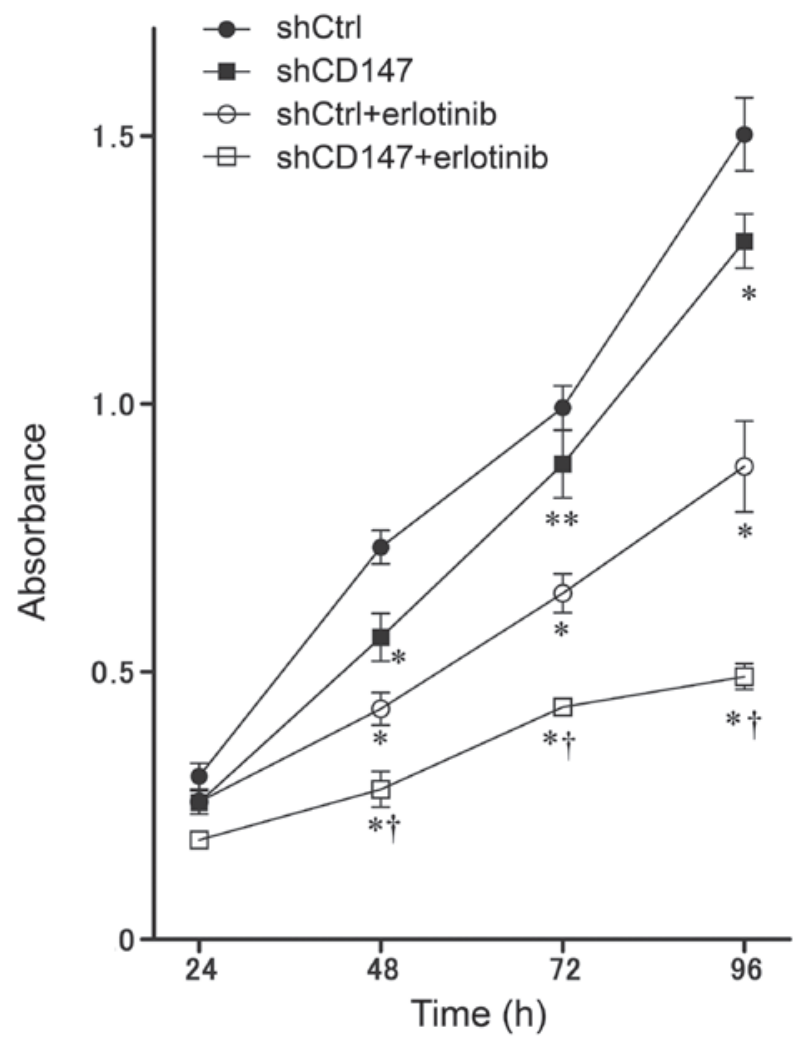

Figure 4. Additive growth inhibition of A375 human malignant melanoma cells by CD147 knockdown (shCD147), treatment with erlotinib (an EGFR inhibitor), or the two techniques in combination. Inhibition of growth was measured using the MTT assay, and the absorbances at $570 \mathrm{~nm}$ and $630 \mathrm{~nm}$ were recorded for test and reference wavelengths, respectively. Growth inhibition was significantly lower in shCD147 cells than control (shCtrl) cells. Erlotinib significantly inhibited the growth of shCtrl cells. Growth inhibition by erlotinib was significantly higher in shCD147 cells than shCtrl cells. ${ }^{*} \mathrm{P}<0.01$ and ${ }^{* *} \mathrm{P}<0.05$ vs. shCtrl; ${ }^{\dagger} \mathrm{P}<0.01$ vs. shCtrl plus erlotinib. MTT, 3-(4,5-dimethylthiazol-2-yl)-2,5-diphenyltetrazolium bromide; EGFR, epidermal growth factor receptor; CD147, cluster of differentiation 147; sh, short hairpin.

Raf-MEK-ERK pathway plays a significant role in oncogenesis. The pathway is activated by either ligand-dependent stimulation of membrane-associated receptor tyrosine kinases or by ligand-independent mechanisms, such as increased expression or mutational activation of Raf. The activating mutation of BRAF triggers the sequential activation of MEK and ERK, resulting in constitutive activation of tumor cell proliferation (2). BRAF has emerged as a therapeutic target due to the high incidence of its mutation in MM. The effects of a highly selective BRAF (V600E) kinase inhibitor, vemurafenib, have been previously studied, and preclinical investigations have revealed potent and selective activity in MM cell lines containing the V600E BRAF mutation (22). Higher overall and progression-free survival rates were demonstrated in a phase 3 randomized clinical trial that compared vemurafenib with dacarbazine in 675 previously untreated metastatic MM patients with the BRAF V600E mutation (23). Despite the marked progress in signal-transduction therapy targeting the MAPK cascade, clinical effectiveness has remained limited.

We previously reported a series of studies on the role of CD147 in MM progression (14-17). CD147 is highly expressed on MM cells and plays an important role in cell proliferation, tumor invasiveness, metastasis and angiogenesis by promoting tumor cell glycolysis. The protein facilitates lactate transport in combination with monocarboxylate transporters (MCTs) (17), and is involved in multidrug resistance by regulating the expression of $\mathrm{P}$-glycoprotein ( $\mathrm{P}-\mathrm{gp}$ ) on cell membranes (16). CD147 appears to have chaperone-like functions in the correct folding, trafficking and cell surface expression of membrane proteins such as MCTs and P-gp. These findings strongly implicated CD147 as a possible therapeutic target for MM. In addition to these MAPK pathway-independent functions, CD147 was demonstrated to directly activate ERK. In MDA-MB-231 and MCF7 breast cancer cells, ERK was phosphorylated by CD147 transfection $(18,19)$. As the A375 MM cells harbor the V600E BRAF activating mutation (20), the correlation between CD147 and the RAF-MEK-ERK pathway was examined in the present study. The phosphorylation level of EGFR in A375-shCtrl and A375-shCD147 cells was investigated. EGFR phosphorylation was anticipated to be reduced by CD147 silencing, as CD147 and EGFR promote tumor cell proliferation. Unexpectedly, EGFR phosphorylation was upregulated by CD147 silencing. Following this result, the phosphorylation of MEK, a downstream effector of B-Raf, was examined. In agreement with previous studies $(18,19)$, the present study demonstrated that MEK phosphorylation was inhibited by CD147 silencing.

Wang et al (24) documented that in Xenopus, cdc25C was activated by MEK, and the cdc 25 homologue, cdc25A, interacted with and dephosphorylated EGFR in human Hep3B hepatoma cells (25). Therefore, we hypothesized that the downregulation of MEK by silencing CD147 would lead to the inhibition of cdc25C and the subsequent phosphorylation of EGFR. In support of this hypothesis, the results of the present study demonstrated that cdc $25 \mathrm{C}$ phosphorylation was downregulated by CD147 silencing. The results herein constitute the first documentation of the functional interaction between CD147 and membrane-associated tyrosine kinase receptors. A similar molecular mechanism has been identified in colon cancer harboring the V600E BRAF mutation (26). Unlike $\mathrm{MM}$, colon cancer with the activating mutation is resistant to vemurafenib, a phenomenon attributed to feedback activation of EGFR. When B-Raf was inhibited by vemurafenib, MEK and cdc25C were downregulated, resulting in EGFR activation (26).

The current study demonstrated the additive effects of CD147 silencing and EGFR inhibition on MM cell growth. The results of the present study suggest that the combination of EGFR and CD147 inhibition may be useful for the treatment of BRAF-mutated MM. Anti-EGFR drugs such as erlotinib, gefinitib, cetuximab and panitumumab are clinically available, and translational research studies on the clinical applicability of CD147 are warranted.

\section{References}

1. Chang L and Karin M: Mammalian MAP kinase signalling cascades. Nature 410: 37-40, 2001.

2. Montagut $\mathrm{C}$ and Settleman J: Targeting the RAF-MEK-ERK pathway in cancer therapy. Cancer Lett 283: 125-134, 2009.

3. Hanahan D and Weinberg RA: The hallmarks of cancer. Cell 100: $57-70,2000$. 
4. Davies H, Bignell GR, Cox C, et al. Mutations of the BRAF gene in human cancer. Nature 417: 949-954, 2002.

5. Kanekura T, Miyauchi T, Tashiro M and Muramatsu T: Basigin, a new member of the immunoglobulin superfamily: Genes in different mammalian species, glycosylation changes in the molecule from adult organs and possible variation in the N-terminal sequences. Cell Struct Funct 16: 23-30, 1991.

6. Miyauchi T, Kanekura T, Yamaoka A, Ozawa M, Miyazawa S and Muramatsu T: Basigin, a new, broadly distributed member of the immunoglobulin superfamily, has strong homology with both the immunoglobulin $\mathrm{V}$ domain and the beta-chain of major histocompatibility complex class II antigen. J Biochem 107: 316-323, 1990.

7. Muramatsu T: Basigin: A multifunctional membrane protein with an emerging role in infections by malaria parasites. Expert Opin Ther Targets 16: 999-1011, 2012.

8. Bordador LC, Li X, Toole B, Chen B, Regezi J, Zardi L, Hu Y and Ramos DM: Expression of emmprin by oral squamous cell carcinoma. Int J Cancer 85: 347-352, 2000.

9. Ishibashi Y, Matsumoto T, Niwa M, Suzuki Y, Omura N, Hanyu N, Nakada K, Yanaga K, Yamada K, Ohkawa K, et al: CD147 and matrix metalloproteinase-2 protein expression as significant prognostic factors in esophageal squamous cell carcinoma. Cancer 101: 1994-2000, 2004.

10. Muraoka K, Nabeshima K, Murayama T, Biswas C and Koono M: Enhanced expression of a tumor-cell-derived collagenase-stimulatory factor in urothelial carcinoma: Its usefulness as a tumor marker for bladder cancers. Int J Cancer 55: 19-26, 1993.

11. Nabeshima K, Suzumiya J, Nagano M, Ohshima K, Toole BP, Tamura K, Iwasaki H and Kikuchi M: Emmprin, a cell surface inducer of matrix metalloproteinases (MMPs), is expressed in T-cell lymphomas. J Pathol 202: 341-351, 2004.

12. Polette M, Gilles C, Marchand V, Lorenzato M, Toole B, Tournier JM, Zucker S and Birembaut P: Tumor collagenase stimulatory factor (TCSF) expression and localization in human lung and breast cancers. J Histochem Cytochem 45: 703-709, 1997

13. Sameshima T, Nabeshima K, Toole BP, Yokogami K, Okada Y, Goya T, Koono M and Wakisaka S: Glioma cell extracellular matrix metalloproteinase inducer (EMMPRIN) (CD147) stimulates production of membrane-type matrix metalloproteinases and activated gelatinase A in co-cultures with brain-derived fibroblasts. Cancer Lett 157: 177-184, 2000.

14. Chen X, Lin J, Kanekura T, Su J, Lin W, Xie H, Wu Y, Li J, Chen $\mathrm{M}$ and Chang J: A small interfering CD147-targeting RNA inhibited the proliferation, invasiveness, and metastatic activity of malignant melanoma. Cancer Res 66: 11323-11330, 2006.

15. Kanekura T, Chen X and Kanzaki T: Basigin (CD147) is expressed on melanoma cells and induces tumor cell invasion by stimulating production of matrix metalloproteinases by fibroblasts. Int J Cancer 99: 520-528, 2002.
16. Kanekura T and Chen X: CD147/basigin promotes progression of malignant melanoma and other cancers. J Dermatol Sci 57: 149-154, 2010.

17. Su J, Chen X and Kanekura T: A CD147-targeting siRNA inhibits the proliferation, invasiveness, and VEGF production of human malignant melanoma cells by down-regulating glycolysis. Cancer Lett 273: 140-147, 2009.

18. Marieb EA, Zoltan-Jones A, Li R, Misra S, Ghatak S, Cao J, Zucker S and Toole BP: Emmprin promotes anchorage-independent growth in human mammary carcinoma cells by stimulating hyaluronan production. Cancer Res 64: 1229-1232, 2004.

19. Yang JM, O'Neill P, Jin W, Foty R, Medina DJ, Xu Z, Lomas M, Arndt GM, Tang Y, Nakada M, et al: Extracellular matrix metalloproteinase inducer (CD147) confers resistance of breast cancer cells to Anoikis through inhibition of Bim. J Biol Chem 281: 9719-9727, 2006.

20. Eskandarpour M, Kiaii S, Zhu C, Castro J, Sakko AJ and Hansson J: Suppression of oncogenic NRAS by RNA interference induces apoptosis of human melanoma cells. Int $J$ Cancer 115: 65-73, 2005

21. Xie W, Xie H, Liu F, Li W, Dan J, Mei Y, Dan L, Xiao X, Li J and Chen X: Propranolol induces apoptosis of human umbilical vein endothelial cells through downregulation of CD147. Br J Dermatol 168: 739-748, 2013.

22. Sala E, Mologni L, Truffa S, Gaetano C, Bollag GE and Gambacorti-Passerini C: BRAF silencing by short hairpin RNA or chemical blockade by PLX4032 leads to different responses in melanoma and thyroid carcinoma cells. Mol Cancer Res 6: 751-759, 2008.

23. Chapman PB, Hauschild A, Robert C, Haanen JB, Ascierto P, Larkin J, Dummer R, Garbe C, Testori A, Maio M, et al; BRIM-3 Study Group: Improved survival with vemurafenib in melanoma with BRAF V600E mutation. N Engl J Med 364: 2507-2516, 2011.

24. Wang R, He G, Nelman-Gonzalez M, Ashorn CL, Gallick GE, Stukenberg PT, Kirschner MW and Kuang J: Regulation of Cdc25C by ERK-MAP kinases during the G2/M transition. Cell 128: 1119-1132, 2007.

25. Wang Z, Wang M, Lazo JS and Carr BI: Identification of epidermal growth factor receptor as a target of $\mathrm{Cdc} 25 \mathrm{~A}$ protein phosphatase. J Biol Chem 277: 19470-19475, 2002.

26. Prahallad A, Sun C, Huang S, Di Nicolantonio F, Salazar R, Zecchin D, Beijersbergen RL, Bardelli A and Bernards R: Unresponsiveness of colon cancer to BRAF (V600E) inhibition through feedback activation of EGFR. Nature 483: 100-103, 2012. 